\title{
Ab Initio Determination of the (100) Surfaces Phonon Dispersions of the SiC
}

\author{
J. S. Soares and H. W. Leite Alves \\ Departamento de Ciências Naturais, Universidade Federal de São João del Rei, \\ Caixa Postal 110, 36300-000 São João del Rei-MG, Brazil
}

Received on 4 April, 2005

\begin{abstract}
In this work, we presented our preliminary ab initio results for the vibrational modes and the phonon frequencies of the $\mathrm{SiC}(100)$ surfaces. Our results are in good agreement with the available experimental data whenever this comparison is possible. For the accepted models of the C-terminated surfaces in the $\mathrm{c}(2 \times 2)$ reconstruction, while in the bridge-dimer model there is an acetylene-like vibrational $A_{1}$ mode at $2031 \mathrm{~cm}^{-1}$, which is infrared active, in the staggered-dimer model, there is a Füchs-Kliewer (FK) mode at $1328 \mathrm{~cm}^{-1}$, which is experimentally detected. For Si-terminated surfaces in the $\mathrm{p}(2 \times 1)$ reconstruction, instead, no FK was obtained, in contradiction with the HREELS experimental results for the Si-terminated surfaces, but they are in consonance with the fact that this surface should be described by a $(3 \times 2)$ or more complex models.
\end{abstract}

Keywords: Ab initio results; Vibrational modes; SiC (100)

\section{INTRODUCTION}

A great amount of efforts is spent to understand the growth mechanism of cubic GaN (and its alloys), which is the prototype material for the optoelectronic devices operating on the visible part of the electromagnetic spectra. Many substrates were employed and, recently, (100) GaAs or SiC substrates were used with relative success $[1,2]$.

Moreover, $\mathrm{SiC}$ is also the ideal material for the fabrication of semiconductor devices operating under extreme conditions [3]. So, the complete knowledge of their (100) surfaces properties is a key for both successful growth of the III-Nitrides as well as for the preparation of the high-temperature microelectronic devices.

However, while the (100) surface electronic structures and structural properties of these materials were extensively studied $[4,5]$, the amount of knowledge on their dynamical properties are rather scarce: only High-resolution electron energyloss spectroscopy (HREELS) data were reported which optical Füchs-Kliewer (FK) phonon modes, that are completely determined by bulk properties, were characterized in these experiments $[6,7]$.

It is well known that the evaluation of dynamical properties of the solids, and of their surfaces, is a powerful tool to give a complete macroscopic characterization of the studied systems and, also, to check the accepted models [8]. So, in this work, by using the Density Functional Theory, within the local density approximation (LDA), based on the plane-wave pseudopotential method together with the slab-supercell description (ABINIT code [9]), we have calculated, ab initio, the phonon dispersions of the C-rich $\mathrm{SiC}(100)$ surfaces, in the $\mathrm{c}-(2 \times 2)$ and $\mathrm{p}(2 \times 1)$ reconstruction patterns, and of the Si-rich $\mathrm{SiC}(100)$ ones, in the $\mathrm{p}(2 \times 1)$ reconstruction model. We have used the Troullier-Martins pseudopotentials in the calculations (obtained with the fhi98PP code [10]), and the phonon dynamics were obtained by means the adiabatic perturbation density functional theory, following our recipe used to describe phonons in GaAs (100) surfaces [11]. In the calculations, the slab supercells were build up of 5 atomic layers and a vacuum region equivalent of 5 atomic layers, and also, a
(4 4 2) Monkhorst-Pack mesh was used to sample the surface Brillouin zone. Details about the (100) surface equilibrium properties of both terminations of $\mathrm{SiC}$ were described in our previous work [12].

\section{SI-TERMINATED SURFACES}

In Fig. 1, we show the phonon dispersion of the Siterminated $\mathrm{SiC}(100)$ surface in the $\mathrm{p}(2 \times 1)$ reconstruction pattern, whose crystalline symmetry is $\mathrm{P}_{m}$. From Fig. 1, we found that there are, besides the Rayleigh mode (RW) (which arrives at $\bar{J}$ point with $103.2 \mathrm{~cm}^{-1}$, and at $\bar{K}$ point with 343 $\mathrm{cm}^{-1}$ ), other three important $\mathrm{Si}$-dimer vibrational modes located in the frequency gap: one mode with $\mathrm{A}^{\prime \prime}$ character at $710 \mathrm{~cm}^{-1}$ (at $\bar{\Gamma}$ point), other $\mathrm{A}^{\prime \prime}$ mode at $562 \mathrm{~cm}^{-1}$ (at $\bar{J}^{\prime}$ point), and one mode with A' symmetry at $730 \mathrm{~cm}^{-1}$ (also at $\bar{\Gamma}$ point). It is interesting to remark that, in the calculated atomic displacement patterns of the modes at $\bar{\Gamma}$ point, the Si atoms vibrates parallel to the surface, while at the $\bar{J}^{\prime}$ point, the $\mathrm{Si}$ atoms vibrates perpendicular to the surface, as depicted in Fig. 2, which we show the displacement pattern of the A" modes at both the $\bar{\Gamma}$ and $\bar{J}^{\prime}$ points of the Brillouin zone.

However, no FK modes were obtained in our calculations for the Si-terminated surfaces: these modes are characterized by vibration of surface atoms parallel to the surface and their frequencies are directly related to the TO bulk mode (which, from our bulk calculations, it was evaluated as $\left.977 \mathrm{~cm}^{-1}\right)$. As the HREELS data on the Si-terminated SiC (100) surfaces show this mode at $936 \mathrm{~cm}^{-1}$ [6], and we did not found this mode in our results for the $\mathrm{p}(2 \times 1)$ reconstruction pattern, this arrangement of surface atoms can be completely ruled out as a model for the Si-terminated surfaces, once it is dominated by weak bonded Si-dimers [12] not observed in the LEED experiments [4]. Instead, we believe that a $(3 \times 2)$ or more complex reconstruction patterns, should be the models that can describe them [13]. Calculations for the Si-terminated $\mathrm{SiC}(100)$ surfaces within the $(3 \times 2)$ reconstruction pattern to check the presence of the observed FK mode are currently under way. 


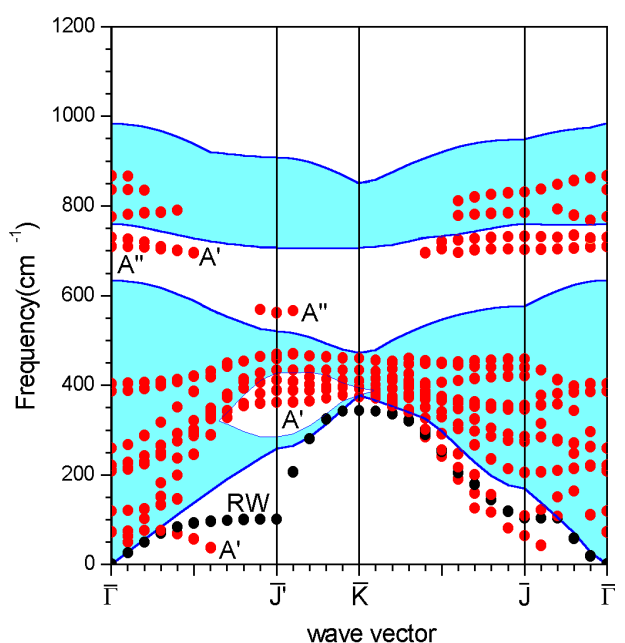

FIG. 1: Calculated phonon dispersion for the Si-terminated $\mathrm{SiC}$ (100) $(2 \times 1)$ surface. The gray circles denote the surface modes, the black ones the Rayleigh wave (RW), while the hatched area are the projected bulk results.

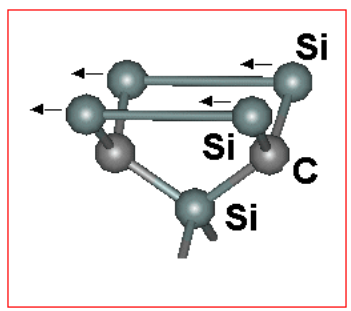

(a)

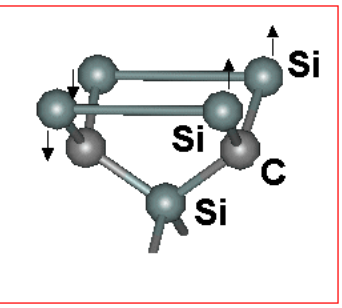

(b)
FIG. 2: Displacement pattern of the surface atoms for the gap A" mode at: (a) $\bar{\Gamma}$ point; (b) $\bar{J}^{\prime}$ point.

\section{C-TERMINATED SURFACES}

We show in Fig. 3, the phonon dispersion of the Cterminated $\mathrm{SiC}(100)$ surface within the $\mathrm{p}(2 \times 1)$ reconstruction pattern, and this system has the $\mathrm{P}_{m m 2}$ crystalline symmetry. From Fig. 3, we found that there are, besides the RW (the lowest frequency mode, which arrives only at the $\bar{J}$ point with $58.9 \mathrm{~cm}^{-1}$ ), other five important $\mathrm{C}$-dimer vibrational modes were obtained: three modes with $\mathrm{A}_{2}$ character (the first at 748 $\mathrm{cm}^{-1}$, the second at $705 \mathrm{~cm}^{-1}$ and the third at $656 \mathrm{~cm}^{-1}$ ) and two $\mathrm{A}_{1}$ modes (one at $701 \mathrm{~cm}^{-1}$ and the other at 1139 $\mathrm{cm}^{-1}$, respectively). The calculated high frequency $\mathrm{A}_{1}$ mode is known as the FK one.

It is interesting to note, from the symmetry selection rules, that while all the calculated vibrational modes are Raman active, only the FK-like mode $A_{1}$ are infrared active. However, none of these kind of experimental results were reported in the literature. Also, we would like to remark that the RW is not present at all k-points of the surface Brillouin zone. As there

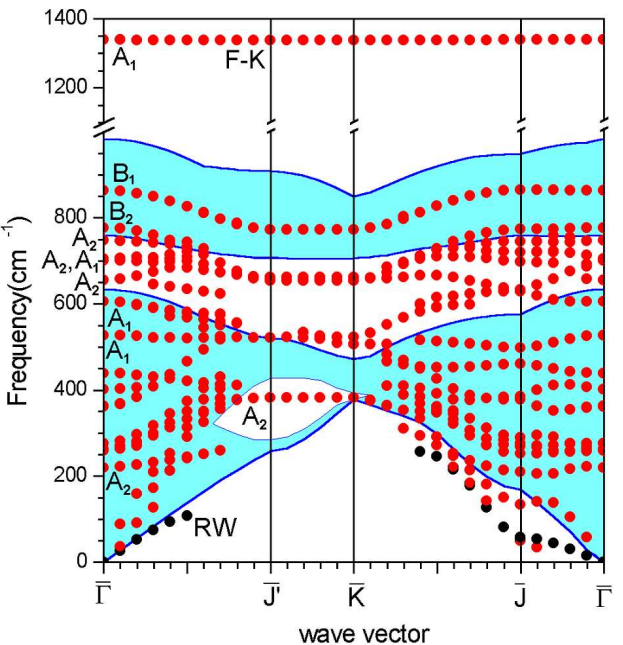

FIG. 3: Same as Fig. 1 for the C-terminated SiC (100) $(2 \times 1)$ surface. Here F-K denotes the Füchs-Kliewer modes.

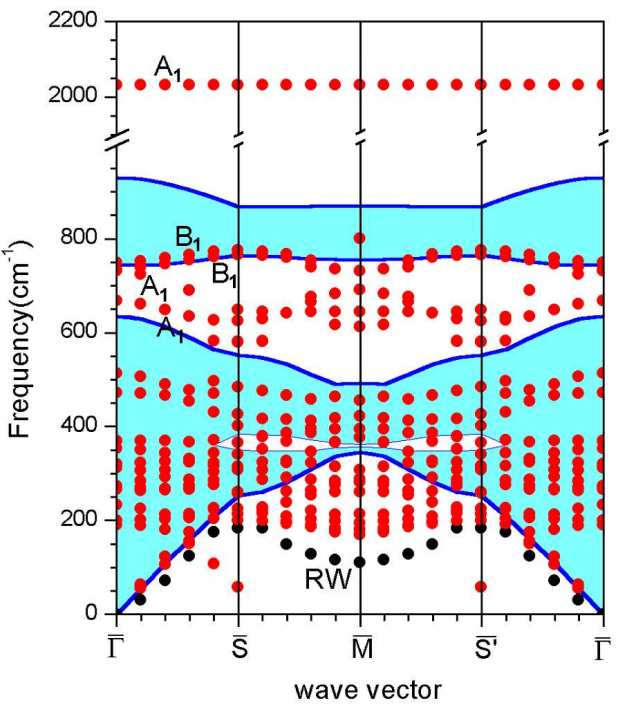

FIG. 4: Same as Fig. 1 for the C-terminated SiC (100) c $(2 \times 2)$ surface in the BDM model.

are no references about this fact reported in the literature, we can infer, from our results, that this is an indication of the low stability of this reconstruction pattern, as already verified in our recent total energy calculations [12].

In Fig. 4, we show the phonon dispersion of the C-rich SiC $(100) c(2 \times 2)$ surface within the Bridge-Dimer Model (BDM), which also has the $\mathrm{P}_{m m 2}$ crystalline symmetry. From Fig. 4, besides the RW (which arrives at $\bar{S}$ point with 185.6 $\mathrm{cm}^{-1}$, and at $\bar{M}$ point with $111.5 \mathrm{~cm}^{-1}$ ), we have detected that there is an $A_{1}$ mode at $2031 \mathrm{~cm}^{-1}$, infrared active, with similar character of $\sigma_{G}$ vibrational mode of the acetylene molecule (from experimental infrared data: $1974 \mathrm{~cm}^{-1}$ [14]), which deals with the stretch of $\mathrm{C}-\mathrm{C}$ triple bonds. We believe that 
this mode is the signature of the BDM, once no FK mode was obtained. Moreover, other four important $\mathrm{C}$-dimer vibrational modes were obtained: two modes with $\mathrm{A}_{1}$ character (one at $668 \mathrm{~cm}^{-1}$ and the other at $732 \mathrm{~cm}^{-1}$ ) and two $B_{1}$ modes (one at $735 \mathrm{~cm}^{-1}$ and the other at $\left.749 \mathrm{~cm}^{-1}\right)$. These modes are all Raman active and only the $A_{1}$ modes are infrared active, by the selection rules.

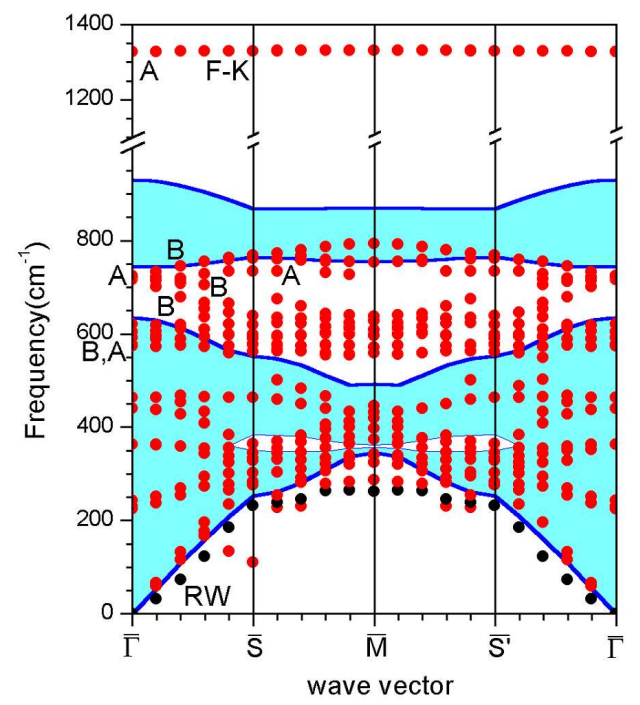

FIG. 5: Same as Fig. 1 for the C-terminated $\mathrm{SiC}(100) \mathrm{c}(2 \times 2)$ surface in the SDM model.

We show, in Fig. 5, the phonon dispersion of the C-rich $\mathrm{SiC}(100) \mathrm{c}(2 \times 2)$ surface within the Staggered-Dimer Model (SDM), which has the $\mathrm{P}_{2}$ crystalline symmetry. Also in Fig. 5 , we have noted that, besides the RW (which arrives at $\bar{S}$ and $\bar{S}^{\prime}$ points of the Brillouin zone with $232.7 \mathrm{~cm}^{-1}$ ), other four Cdimer vibrational modes were obtained: two modes with $\mathrm{A}$ character (one at $720 \mathrm{~cm}^{-1}$ and the other at $1328 \mathrm{~cm}^{-1}$ ), and two B modes (one at $716 \mathrm{~cm}^{-1}$, and the other at $725 \mathrm{~cm}^{-1}$ ). All modes in this system are both infrared and Raman active, and the higher A mode have the FK character.

It is interesting to note that the reported HREELS data on the C-terminated SiC surfaces [6] describes only the optical FK phonon modes. The experimental data of Balster et al. show this mode located at $958 \mathrm{~cm}^{-1}$, which is $38 \%$ below our obtained values for the SDM. Considering that the total energy difference between BDM and SDM are small $[5,12]$, and that the experiment were done at room temperature [6], we can infer, from our results, that Balster et al. were dealing with the SDM model in their C-terminated SiC $(100) c(2 \times 2)$ surface.

\section{CONCLUSIONS}

In summary, we have studied the vibrational modes of the $p(2 \times 1)$ reconstruction model for the Si-terminated surfaces, and for $\mathrm{p}(2 \times 1)$ and $\mathrm{c}(2 \times 2)$ reconstruction patterns within both BDM and SDM models for the C-terminated ones. For the C-terminated surfaces, while in the BDM there is an acetylene-like vibrational $A_{1}$ mode at $2031 \mathrm{~cm}^{-1}$, which is infrared active, in the SDM, there is a FK mode at $1328 \mathrm{~cm}^{-1}$, in agreement with the experimental results. However, for Siterminated surfaces in the $\mathrm{p}(2 \times 1)$ reconstruction, no FK mode was obtained, in contradiction with the HREELS experimental results for this surface. We hope that our results give guidelines for future experiments on this subject, and a complete description of our obtained results will be published soon in another publication.

\section{Acknowledgements}

J. S. Soares acknowledges the Brazilian Program $\mathrm{PIBIC} / \mathrm{CNPq}$ for the financial support. This work was also supported by the MCT/CNPq/PRONEX project No. 662105/98, Brazil.
[1] D. Troost, H. -U. Baier, A. Berger and W. Mönch, Surf. Sci. 242, 324 (1991).

[2] H. Okumura, H. Hamaguchi, G. Feuillet, Y. Ishida and S. Yoshida, Appl. Phys. Lett. 72, 3056 (1998).

[3] R. S. Kern and R. F. Davies, Mater. Sci. Eng. B46, 240 (1997).

[4] V. M. Bermudez, Phys. Stat. Sol. (b)202, 447 (1997), and references therein.

[5] J. Pollmann and P. Krüger, J. Phys. Condens. Matter 16, S1659 (2004), and references therein.

[6] T. Balster, V. M. Polyakov, H. Ibach, and J. A. Schaefer, Surf. Sci. 416, 177 (1998).

[7] H. Nienhaus, V. van Elsbergen, and W. Mönch, Eur. Phys. J. B9, 179 (1999).

[8] S. Baroni, S. de Gironcoli, A. Dal Corso, and P. Giannozzi, Rev.
Mod. Phys. 73, 515 (2001).

[9] X. Gonze et al., Comput. Mater. Sci. 25, 478 (2002), and references therein.

[10] M. Fuchs and M. Scheffler, Comput. Phys. Commun. 119, 67 (1999).

[11] H. W. Leite Alves, J. L. A. Alves, A. M. Santos, L. M. R. Scolfaro, and J. R. Leite, Braz. J. Phys. 34, 617 (2004).

[12] J. S. Soares and H. W. Leite Alves, Microelectron. J. 36, 998 (2005).

[13] P. Soukiassian, Mater. Sci. Eng. B96, 115 (2002); V. Derycke et al., Nature Materials 2, 253 (2003).

[14] G. B. B. M. Sutherland, Phys. Rev. 43, 883 (1933); M. Herman et al., J. Phys. Chem. Ref. Data 32, 921 (2003). 